\title{
Bedside Evaluation for Dysphagia in Jordan
}

\author{
Original \\ Article \\ Jehad A. Alaraifi, Wesam B. Darawsheh ${ }^{2}$, and Yaser S. Natour ${ }^{3}$ \\ ${ }^{1}$ Department of Hearing and Speech Sciences, ${ }^{2}$ Occupational Therapy, School of \\ Rehabilitation Sciences, the University of Jordan, ${ }^{3}$ Department of Audiology and Speech \\ Pathology, Al-Ahliyya Amman University, \& Department of Hearing and Speech Sciences \& \\ Disorders, School of Rehabilitation Sciences, the University of Jordan. Jordan.
}

\begin{abstract}
Aim: To explore the implemented procedures of bedside evaluation for patients with dysphagia by Jordanian healthcare professionals.

Patients and Methods: A cross-sectional descriptive study carried out in five public and five private healthcare institutions in the central and northern areas of Jordan (Amman and Jerash). Participants were healthcare professionals from different disciplines. They were asked to complete a self-administered questionnaire concerning the implemented bedside evaluation procedures for dysphagia in their facilities.

Results: A total of eighty-six participants completed the survey. Thirty-nine (45.3\%) were males and $47(54.6 \%)$ were females (age range 22 - 55; mean: 31.6 years). When asked about beside evaluation procedures, 63 (73.3\%) of the participants reported oral examination as a common bedside evaluation procedure followed by case history reports $(60 ; 69.8 \%)$, then observing patients' complaint/report of gastroesophageal reflux disorder (44; 51.2\%). Compared with other professionals, speech language pathologists had more knowledge about procedures involved in bedside evaluation for dysphagia conditions.

Conclusion: Healthcare professionals' knowledge about procedures involved in dysphagia bedside evaluation in Jordan is insufficient.

Key Words: Bedside evaluation, dysphagia, healthcare professionals, rehabilitation.

Received: 28 August 2020, Accepted: 1 October 2020

Corresponding Author: Wesam B. Darawsheh, Associate Professor, Department of Occupational Therapy, School of Rehabilitation Sciences, the University of Jordan, Queen Rania Al Abdallah St. Amman Jordan, Tel.: +962-6-5355000, E-mail:w.darawsheh@ju.edu.jo
\end{abstract}

ISSN: 2090-0740, 2021, Vol.22

\section{INTRODUCTION}

Dysphagia is a medical condition caused by possible structural and/or functional problems of the upper gastrointestinal tract ${ }^{[1,3]}$. Severe dysphagia may cause several medical complications including aspiration, pneumonia and/or malnutrition ${ }^{[3,4]}$. Several studies investigated symptoms of dysphagia, such as coughing after swallowing, dysphonia, abnormal gag reflux, dysarthria, or voice change after swallow ${ }^{[1,3,4]}$. However, several dysphagia conditions can be asymptomatic or "silent" $"[5,6]$.

For better diagnoses of dysphagia, the evaluation protocol includes various subjective tests and instrumental techniques, e.g. modified barium swallow testing (videofluoroscopy) and videoendoscopy ${ }^{[7,8]}$. Noninstrumental evaluation methods include questionnaires, water swallow tests, and clinical bedside examination ${ }^{[7,8]}$. An example of questionnaires is the dysphagia handicap index ${ }^{[9]}$. Concerning water screening tools, most common are the Yale swallow protocol ${ }^{[10]}$ and the GUGGING swallow test $\left.{ }^{[11,12}\right]$. Such questionnaires and tools were translated into different languages including Arabic ${ }^{[9,12]}$.
Several studies targeted several dysphagia evaluation procedures, including bedside evaluation. Dysphagia bedside evaluation helps tailor treatment plans to patient needs and assess treatment outcomes ${ }^{[3,8,13,14]}$. There are several procedures involved in Dysphagia bedside evaluation which aim to investigate the severity and possible etiologies of Dysphagia. However, there are contentions concerning which of these procedures need to be implemented. Shipley and McAfee ${ }^{[13]}$ stated that bedside evaluation needs to include procedures such as testing cognitive skills, documenting the current medical status of the patient, evaluation of feeding, dietary and laryngeal conditions. Vogels, Cartwright, and Cocks ${ }^{[15]}$ conducted a study that utilized a questionnaire followed by interviews to explore the procedures involved in bedside evaluation in Australia. Babani and Hattiangadi ${ }^{[16]}$ conducted a study in south Mumbai that aimed to construct a bedside examination protocol to evaluate and grade dysphagia conditions. Results from the literature generally showed that motor evaluative procedures were predominantly used rather than sensory evaluative procedures. 
Generally, the literature shows that dysphagia bedside evaluation includes two main elements; the first is case history and the second is clinical examination. Case history involves collecting information concerning any history of previous pneumonia, the types of difficult food/ difficult textures, problems with biting, chewing, drooling, and reduced tongue movement/strength/ range of movement $t^{[1,13,15,17]}$. Information is also collected concerning reduced oral sensations, any pocketing of food, nasal regurge of food, coughing or chocking during the meal (before/during / after) the swallow. Case history also involves collecting information concerning any newly developed eating habits; such as the formation of smaller bolus formation, dry swallows, assuming certain postures, and taking longer mealtimes ${ }^{[1,13,15,17]}$.

Clinical examination of dysphagia bedside evaluation includes procedures such as oral examination, vocal tract examination, neurological examination, and observation during trial feeding. Additionally, clinicians may observe the presence of abnormal behaviors, such as coughing, choking, voice change before, during or after swallowing which might constitute a sign of swallowing difficulties ${ }^{[13,18,19]}$. During trials of feeding, the patient is introduced to different food consistencies (e.g. liquid, puree, solid); and dry swallows are also used ${ }^{[16]}$. It also involves observing any swallowing-difficulties the patient may exert while swallowing. The process may include physiological examination of swallowing and oral sensory motor tasks in addition to cognitive and communication evaluation $^{[7,20]}$.

Due to the complexity of dysphagia conditions, the dysphagia evaluation protocol should be carried out by multiple healthcare professionals where any further referrals are needed, they should be made to Speech Language Pathologists (SLPs) ${ }^{[21]}$. The team of professionals includes, but is not limited to, occupational therapists, physical therapists, social workers, nurses, nutritionist/dietician, and medical doctors from different specializations $^{[21,22]}$. Darawsheh et al. ${ }^{[22]}$ conducted a study of 22 healthcare professionals to explore the rehabilitation services provided for dysphagia patients in medical settings in Jordan. The results of that study showed that healthcare professionals demonstrated limited knowledge of their role with dysphagia conditions. Results also showed that the provision of rehabilitation services was minimal and secondary to the provision of other medical services. Darawsheh et al. ${ }^{[22]}$ showed that professional practices in dysphagia assessment and rehabilitation are generally not well supported by evidence-based practice other than studies conducted in Western countries. The current study, therefore, aims to investigate the procedures involved in bedside evaluation for dysphagia conditions in Jordan, and professionals involved in the implementation of these procedures.

\section{PATIENTS AND METHODS:}

\subsection{Study Design}

The study utilized a cross sectional-descriptive design and was conducted in five public and five private health institutions in the central(Amman) and northern (Jerash) areas of Jordan.

\subsection{Participants and Study Setting}

Participants were healthcare professionals who were nurses, nutritionists, psychologists, occupational therapists (OTs), physiotherapists (PTs), social workers and speech language pathologist (SLPs). Only professionals who reported dysphagia conditions caseloads participated in the study.

Prior to interviewing the targeted professionals, an ethical approval was obtained from the Institutional Review Board (IRB) at the Deanship of Scientific Research, the University of Jordan. Professionals signed an informed consent form prior to participation. Participants then completed a semi-structured questionnaire (appendix 1) that included both multiple choice and informational questions. An example was "what are the procedures involved as part of bedside evaluation in your facility?". The purpose of such open-ended questions was to further explore the professionals' knowledge about the nature of bedside evaluation conducted for patients with dysphagia in their institutions.

\subsection{Data Analysis}

Data were analyzed using SPSS Statistics for Windows, Version 25.0 released (IBM Corporation, USA). Categorical variables were compared using the Chi-square test and, Fisher's exact test. Significance level was preset as $p<0.05$.

\section{RESULTS:}

A total of 86 participants completed the survey. Thirtynine $(45.3 \%)$ were males and $47(54.6 \%)$ were females (age range $22-55$; mean 31.6 years). (Table 1) lists the demographics of participants).

Table 1: Participants' Demographic Information.

\begin{tabular}{ll}
\hline Practitioners & $\mathrm{n}(\%)$ \\
\hline Gender & $39(45.3)$ \\
Males & $47(54.6)$ \\
Females & \\
Education & $8(9.3)$ \\
Diploma & $70(81.4)$ \\
B.A./ BSc & $5(5.8)$ \\
M.A./ MSc & $3(3.5)$ \\
PhD &
\end{tabular}


Occupation

\begin{tabular}{ll} 
Medical doctor & $21(24.4)$ \\
Nurse & $35(40.7)$ \\
Nutritionist & $3(3.5)$ \\
Psychologist & $4(4.7)$ \\
SLP & $7(8.1)$ \\
Occupational Therapist & $6(7.0)$ \\
Physiotherapist & $6(7.0)$ \\
Social Worker & $4(4.6)$ \\
\hline
\end{tabular}

Table 2 shows that professionals listed multiple evaluation procedures used in bedside evaluation. The most frequently listed procedures were conducting an oral examination 63 (73.3\%), taking a case history 60 (69.8\%), exploring signs for gastroesophageal reflux disorder (GERD) 44 (51.2\%), checking gag reflex 41 (47.7\%), testing different food textures $39(45.3 \%)$, and meal observation 37 (43\%).

The most reported procedures conducted by SLPs were the oral exam $7(100 \%)$, case history $7(100 \%)$, meal observation $7(100 \%)$, and testing different food textures 7 (100\%). Significant differences were found between SLPs and other healthcare professionals' choice of procedures as related to food texture $(p<0.01)$, food temperature $(p<0.05)$, and meal observation $(p<0.01)$.

Table 2: Reported Procedures of Bedside Evaluation.

\begin{tabular}{lcccc}
\hline Procedure & All professionals $(\mathrm{n}=86)$ & SLPs $(\mathrm{n}=7)$ & Excluding SLPs $(\mathrm{n}=79)$ & P value \\
\hline & $\mathrm{n}(\%)$ & $\mathrm{n}(\%)$ & $\mathrm{n}(\%)$ & $32(40.5)$ \\
Test different food textures & $39(45.3)$ & $7(100)$ & $16(20.3)$ & $0.003^{* *}$ \\
Test different food temperatures & $20(23.3)$ & $4(57.1)$ & $20(25.3)$ & $0.048^{*}$ \\
Test different food tastes & $24(27.9)$ & $4(57.1)$ & $53(67.1)$ & 0.091 \\
Take case history & $60(69.8)$ & $7(100)$ & $56(70.9)$ & 0.090 \\
Conduct an oral exam & $63(73.3)$ & $7(100)$ & $30(38.0)$ & 0.182 \\
Meal observation & $37(43.0)$ & $7(100)$ & $27(34.2)$ & $0.002^{* *}$ \\
Chest rattle observation & $30(34.9)$ & $3(42.9)$ & $30(38.0)$ & 0.690 \\
Observe mouth secretions & $34(39.5)$ & $4(57.1)$ & $39(49.4)$ & 0.420 \\
Check gastroesophageal reflux & $44(51.2)$ & $5(71.4)$ & $38(48.1)$ & 0.430 \\
Check Gag reflex & $41(47.7)$ & $3(42.9)$ & 1.000 \\
\hline
\end{tabular}

Note. $*$ Significant at $p<0.05$ level, $* *$ Significant at $p<0.01, * * *$ Significant at 0.001 level.

Overall, the mean number of bedside evaluation procedures reported by the other healthcare professionals was (4.2); while the mean number of total bedside evaluation procedures identified by SLPs group was (7.2).

\section{DISCUSSION}

According to the results, most participants listed oral exam as the most common bedside evaluation procedure, followed by case history reports. These results were expected because conducting an oral exam and formulating a case history report are common practices performed with all patients as part of the documentation process and constructing the patient profiles ${ }^{[1,13,15]}$. In fact, Scharitzer et al. ${ }^{[17]}$ stated that it is common practice for health professionals to conduct full comprehensive case histories to reach reliable decisions.

A total of 41 participants $(47.7 \%), 3$ SLPs and 38 other professionals, listed gag reflex an integral procedure of the dysphagia bedside evaluation.
This may be due to the common association made between the absence of normal gag reflex and the presence of swallowing problems ${ }^{[23]}$. In contrast, other studies stated that testing gag reflex does not help in predicting the presence of dysphagia ${ }^{[24]}$. As such, controversy regarding this issue was reflected in the participants' responses ${ }^{[19,25]}$.

According to the findings, all professionals listed meal observation, testing different food textures, temperatures and tastes as part of their procedures. As such, it seems that those professionals conduct food trials during bedside evaluation. It is documented that observing the patient while trying different food textures is related to physiological behavior and the flow of the bolus during swallowing ${ }^{[13,26]}$. However, the application of such a procedure depends on the healthcare professional's clinical judgment/ experience and knowledge, and the patient's tolerance (what the patient can or prefer to eat and what he/she cannot eat ${ }^{[7,13]}$. This may explain why some professionals did not mention food trials as a procedure of bedside evaluation. 
When the results of SLPs were compared with those of other healthcare professionals, different interrelations and interpretations emerged. All participating SLPs listed the following procedures: case history, oral examination, testing different food textures, and meal observation as part of the procedures used in bedside evaluation. The least used procedures by the SLPs were gag reflex testing and chest rattle sounds. Thus, it appears that SLPs did not use gag reflex testing as a predictor of dysphagia. This may be due to the controversies reported by studies investigating testing gag reflex ${ }^{[19,25]}$. Moreover, SLPs did not list chest rattle as another predictor of dysphagia because they might have considered it an independent instrumental technique not as a bedside evaluation procedure ${ }^{[27]}$.

Significant differences were found between SLPs and all other healthcare professionals' choice of procedures in terms of food texture $(p<0.01)$, food temperature $(p<=0.05)$, and meal observation $(p<0.01)$. Examining the means and percentages listed in table 2 revealed that SLPs were less conservative than other healthcare professionals in trying out different food textures and temperatures during bedside evaluation. This may be due to the fact that SLPs are trained specifically in the areas of bedside evaluation and meal observation. In fact, manipulation of food textures and temperatures is an integral part of SLPs training to remediate swallowing problems ${ }^{[27]}$. Moreover, food trials should be administered with caution because it might put the patient at risk if administered inappropriately. This may explain why it was an avoided procedure by other professionals.

The mean number of bedside evaluation procedures conducted by other healthcare professionals was lower than that of the SLPs' group. This indicated that SLPs were more aware of the procedures of bedside assessment compared to other healthcare professionals. This result was expected as the standard training of SLPs must contain training on dysphagia conditions $^{[21]}$.

\section{CONCLUSION}

Based on the results of the current study, there is less knowledge among other healthcare professionals regarding the nature of bedside evaluation for patients with dysphagia as compared to SLPs. Bedside evaluation of dysphagia should be emphasized as part of the education for all healthcare professionals who work with dysphagia conditions. SLPs should have a leading role in interacting with other professionals to optimize the evaluation and intervention processes for patients with dysphagia.

\section{LIMITATIONS OF THE STUDY}

Due to the small sample size, the results of the current study cannot be generalized to the entire population of healthcare professionals in Jordan. It is suggested that further studies be conducted to address bedside evaluation and relate results to different medical conditions.

Another recommendation is the investigation of the use of other non-instrumental methods such as the dysphagia handicap index ${ }^{[9]}$, the Yale swallow protocol $^{[10]}$ and the GUGGING swallow test ${ }^{[11,12]}$ ) for dysphagia evaluation by SLPs in Jordan.

\section{CONFLICT OF INTEREST}

Ethical approval to pursue this study was granted from the deanship of scientific research at the University of Jordan, reference number [10/2016/150].

\section{CONFLICT OF INTEREST}

There are no conflicts of interest.

\section{ACKNOWLEDGEMENTS}

This research was supported by the deanship of scientific research at the University of Jordan.

\section{REFERENCES}

1. Aslam M, Vaezi MF. Dysphagia in the elderly. Gastroenterol Hepato. 2013;9(12):784.

2. Carucci LR, Turner MA. Dysphagia revisited: common and unusual causes. Radiographics. 2015; 35(1):105-22.

3. O'Horo JC, Rogus $\square$ Pulia N, Garcia $\square$ Arguello L, Robbins J, Safdar N. Bedside diagnosis of dysphagia: a systematic review. J Hosp Med. 2015;10(4):256-65.

4. Daniels SK, Ballo LA, Mahoney MC, Foundas AL. Clinical predictors of dysphagia and aspiration risk: outcome measures in acute stroke patients. Arch Phys Med Rehabil. 2000;81(8):1030-3.

5. Løkke A, Dongo LC, Aksglæde KB, Hilberg O. Asymptomatic dysphagia causing recurrent aspiration pneumonia. Case Rep. 2018;2018:bcr-2018.

6. Ramsey D, Smithard D, Kalra L. Silent aspiration: what do we know?. Dysphagia. 2005;20(3):218-25.

7. Carnaby-Mann G, Lenius K. The bedside examination in dysphagia. Phys Med Rehabil Clin N Am. 2008;19(4):747-68. 
8. Maccarini AR, Filippini A, Padovani D, Limarzi M, Loffredo M, Casolino D. Clinical non-instrumental evaluation of dysphagia. Acta Otorhinolaryngol Ital. 2007;27(6):299-305.

9. Farahat M, Malki KH, Mesallam TA, Bukhari M, Alharethy S. Development of the arabic version of dysphagia handicap index (DHI). Dysphagia. 2014;29(4):459-67.

10. Leder SB, Suiter DM. The Yale swallow protocol: an evidence-based approach to decision making. Springer; 2014

11. AbdelHamid A, Abo-Hasseba A. Application of the GUSS test on adult egyptian dysphagic patients. Egypt J Otolaryngol. 2017;33(1):103.

12. Trapl M, Enderle P, Nowotny M, Teuschl Y, Matz K, Dachenhausen A, Brainin M. Dysphagia bedside screening for acute-stroke patients: the Gugging Swallowing Screen. Stroke. 2007;38(11):2948-52.

13. Shipley KG, McAfee JG. Assessment in speechlanguage pathology: A resource manual. Boston: Delmar Cengage Learning; 2016.

14. McAllister S, Kruger S, Doeltgen S, Tyler-Boltrek E. Implications of variability in clinical bedside swallowing assessment practices by speech language pathologists. Dysphagia. 2016;31(5):650-62.

15. Vogels B, Cartwright J, Cocks N. The bedside assessment practices of speech-language pathologists in adult dysphagia. Int $\mathrm{J}$ Speech Lang Pathol. 2015;17(4):390-400

16. Babani ND, Hattiangadi GA. Bedside assessment protocol and grading scale for dysphagia in adults: A preliminary study. J Indian Speech Lang Hear Assoc. 2014;28(1):10.

17. Scharitzer M, Pokieser P, Wagner-Menghin M, Otto F, Ekberg O. Taking the history in patients with swallowing disorders: an international multidisciplinary survey. Abdom Radio. 2017;42(3):786-93.
18. The American Speech-Language-Hearing Association Adult Dysphagia: Assessment. Avaialble from: http:// www.asha.org/PRPSpecific Topic.aspx? folderid $=858$ 9942550\&section $=$ Assessment $[$ Accessed 21st July, 2020].

19. Hassan HE, Aboloyoun AI. The value of bedside tests in dysphagia evaluation. Egyptian J Ear Nose Throat and Allied Sci. 2014;15(3):197-203.

20. Kanna SV, Bhanu K. A simple bedside test to assess the swallowing dysfunction in Parkinson's disease. Ann Indian Acad Neurol. 2014 Jan;17(1):62.

21. The American Speech-Language-Hearing Association. Dysphagia Teams. Avaialble from: https://www. asha.org/Practice-Portal/Clinical-Topics/PediatricDysphagia/Dysphagia-Teams [Accessed 21st July, 2020].

22. Darawsheh, W., Alaraifi, J., \& Natour, Y. (2020). A Pilot Study of the Rehabilitation Services Provided for Patients with Dysphagia in Medical Settings in Jordan. Res Health Sci. 2020;5(2):86-106.

23. Schroeder MF, Daniels SK, McClain M, Corey DM, Foundas AL. Clinical and cognitive predictors of swallowing recovery in stroke. J Rehabil Res Dev. 2006;43(3):301.

24. Leder SB. Gag reflex and dysphagia. Head Neck: J Sci and Spec Head Neck. 1996;18(2):138-41.

25. Ramsey DJ, Smithard DG, Kalra L. Early assessments of dysphagia and aspiration risk in acute stroke patients. Stroke. 2003;34(5):1252-7.

26. Steele CM, Alsanei WA, Ayanikalath S, Barbon CE, Chen J, Cichero JA, Coutts K, Dantas RO, Duivestein J, Giosa L, Hanson B. The influence of food texture and liquid consistency modification on swallowing physiology and function: a systematic review. Dysphagia. 2015;30(1):2-6.

27. Logemann, J. A. (1998). Evaluation and treatment of swallowing disorders. Austin, Tex: PRO-ED; 1998. 


\section{Appendix 1}

The Survey: Bedside Evaluation for Dysphagia in Jordan

الإجر اءات المتبعة في الفحص السريري (Bedside evaluation) لحالات صعوبات البلع في الأردن Bedside Evaluation Procedures for Patients with Dysphagia in Jordan

القسم الأول: معلومات ديمو غر افية First Section: Demographic Information

$$
\begin{aligned}
& \text { العرر: } \\
& \text { Age } \\
& \text { Gender } \\
& \text { Educational Level } \\
& \text { مكان العمل: } \\
& \text { Workplace } \\
& \text { القسم: } \\
& \text { المهنة و التخصص: } \\
& \text { Department }
\end{aligned}
$$

Number of years of experience

القسم الثاني: تقييم حالات صعوبات البلع

Second Section: Dysphagia Evaluation

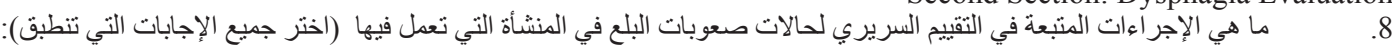
(What swallowing bedside procedures are conducted in your facility (check all that apply فحص الفم

Orawl exam

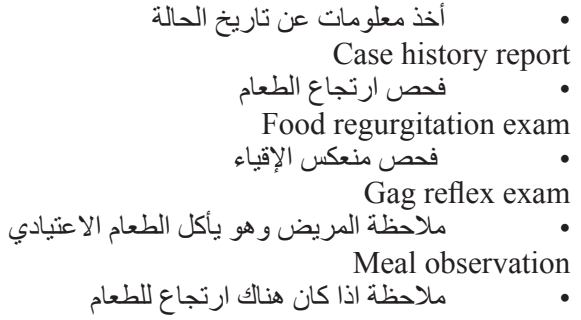

Documenting incidence of food regurgitation

$$
\text { • }
$$
Drooling

تقييم المريض و هو يأكل عدة أنواع من الطعام بقو ام مختلف

Evaluating the effect different foods on swallowing تقيبم المريض و هو يأكل عدة أنو اع من الطعام بمذاقات مختلفة

Evaluating the effect of different food textures and taste on swallowing تقييم المريض وهو يأكل عدة أنو اع من الطعام بدرجات حر ارة مختلفة

Evaluating the effect of different food temperatures on swallowing

هل هناك إجر اءات أخرى متبعة غير المذكورة أعلاه، إذا كان الجو اب تعم فيرجى أن تحدد ذلك.

Are there any additional procedures? If there are, please mention

القسم الثالث: الأخصائيين الذين بقومون بثقييم حالات صعوبات البلع

Third Section: Professional who undertake the evaluation of swallowing disorders 10.

(Who conducts swallowing evaluation in your facility? (check all that apply أخصائي العلاج الوظيفي

Occupational therapis خصائي علاج طبيعي

Physical therapist طبيب

Medical docto ممرض

Nurse

Psychotherapis 


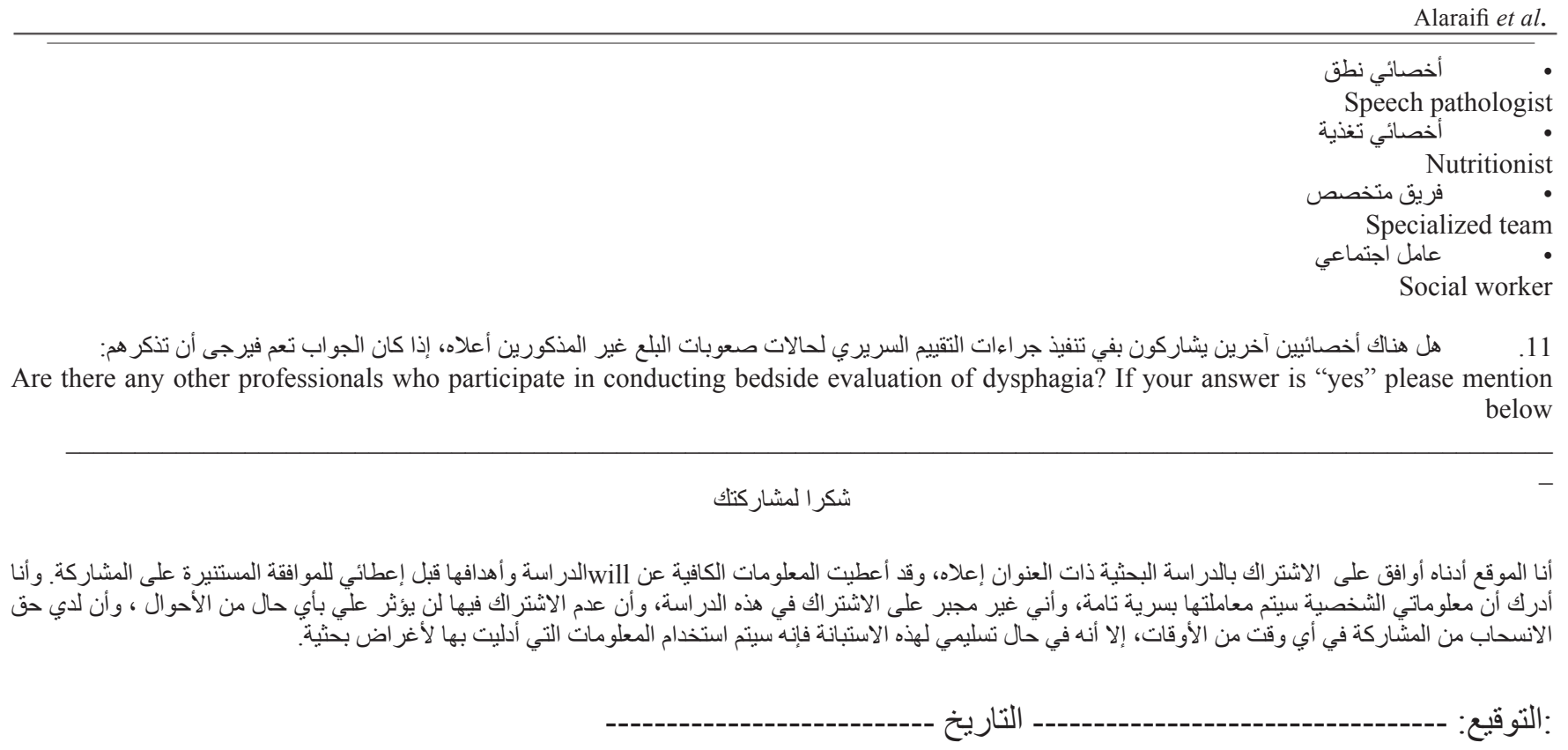

I, the undersigned herein, agree on participating in the research study entitled above. I was provided with sufficient information regarding the study and its goals. I realize that my personal information will be treated with utmost confidentiality. I also understand that I am not obliged to participate in the current study and there will be no consequences whatsoever for not participating in the study. I reserve the right to withdraw form the study any time. I do realize that my questionnaire answers will be used for research purposes only.

Signature:

Date: 